\title{
EL NUEVO FENÓMENO EUROPEO DEL TURISMO DE SALUD: ESTADO DE LA CUESTIÓN
}

\author{
Isabel Casabona Martínez* \\ Manuel Lillo Crespo* \\ $M^{a}$ Dolores Mora Antón** \\ Roberto Galao Malo***
}

*Clínica Vistahermosa. Profesor/a Asociado/a Departamento de Enfermeria.U.A.

Diplomado/a en Enfermería, Licenciado/a en Antropología Social y Cultural.

**Directora de Enfermeria. Clínica Vistahermosa. Diplomada en Enfermería. ***Clínica Vistahermosa. Licenciado Segundo Ciclo de Enfermería. Diplomado en Enfermería.

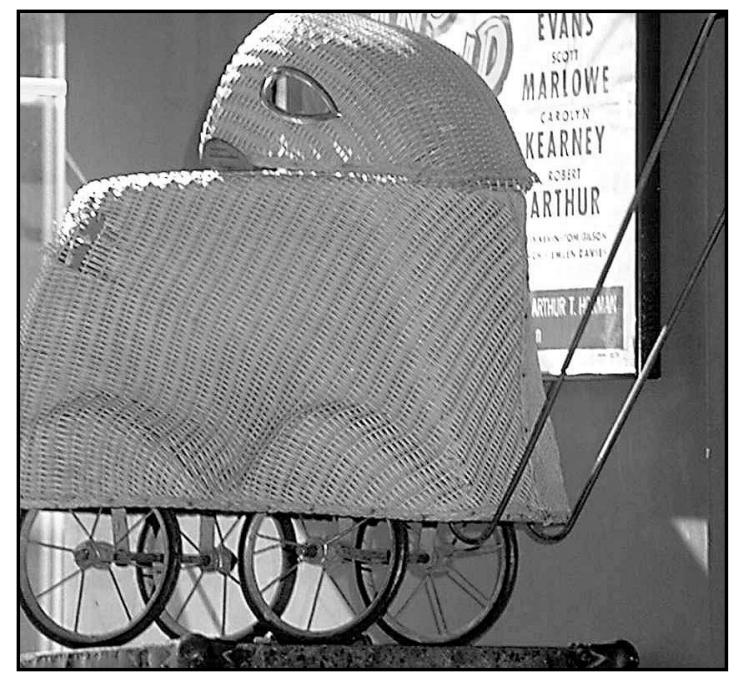

THE NEW EUROPEAN PHENOMENON. HEALTH TOURISM. PRESENT SITUATION

\section{ABSTRACT}

$\mathrm{T}$ The present crisis in health system is something common world wide. This crisis can be well detected when watching waiting lists for surgical care treatments, according to the increasing number of operation processes due to the better present surgical and anesthetic techniques. Also due to the more and more demands from the population. This is a process where finance for certain specific processes emphasizes.

The health systems are looking for alternative ways to diminish their waiting lists as well as to decrease costs. For that reason, the European Union opens its borders to easy a possible traffic of patients among different countries to allow users of a certain Union Estate to move to another one. In some occasions a full team of professionals: doctors and nurses could move from their own European Estate to a different one. But of course, this type of transfers do present certain problems. The native country must guarantee that the care the patient will get in another country will be similar to its own. The receiving country faces the challenge of caring patients with a different culture, a different language and as a consequence different ways for considering health where illness and hospitalization differ as well.

Clinica Vistahermosa - in Alicante - has been pioneer in that sense, in the whole Spain, and within Europe. CV. has agreements and protocols signed with Portugal, U.K. and Holland. As a matter of fact, it has been receiving patients from the later for several years now.

As we have been showing in several studies, there is a new phenomenon we may call "Health Tourism" in Alicante, as patients integrate not only for health techniques but for leisure time as well as they book hotel rooms for post-operative sessions.

Present situation, challenges and consequences of this new phenomenon are presented in this study.

KEY WORDS: health exchanges, administration, transcultural perspectives, cultural competences, satisfaction, quality of care, health tourism 


\section{RESUMEN}

$\mathrm{L}$ a crisis de los sistemas sanitarios es algo común en todos los países del mundo.

Esta crisis se ve especialmente reflejada en las listas de espera para tratamientos de cirugía, en la medida en que el número de intervenciones aumenta debido a las mejoras de las técnicas quirúrgicas y anestésicas así como al incremento de la demanda de la población. Todo esto conlleva que los gastos en estas materias específicas se disparen.

Los sistemas sanitarios vienen buscando alternativas que aceleren las listas y disminuyan los costes. Con este fin, la Unión Europea ha abierto las fronteras al tráfico de pacientes entre países, lo que permite que usuarios de un estado vayan a operarse a otro, en ocasiones con médicos y equipo del propio país de origen. Sin embargo esto plantea ciertos problemas: el país de origen debe garantizar que la asistencia va a ser similar a la que se le prestaría en este, debe elegir el tipo de intervenciones y el tipo de pacientes adecuados y un largo etcétera. El país de destino afronta el reto de cuidar a unos pacientes con una lengua y una cultura diferentes y por lo tanto con unas creencias sobre la salud, la enfermedad y la hospitalización diferentes.

La Clínica Vistahermosa ha sido pionera a este campo en el ámbito español y europeo, gracias a los acuerdos firmados entre nuestro país y otros como Portugal, Gran Bretaña u Holanda. De este último venimos recibiendo pacientes desde hace varios años.

Tal y como venimos reflejando en diferentes estudios, se esta dando un fenómeno nuevo que denominamos "Turismo de Salud" en la provincia de Alicante, puesto que los pacientes realizan actividades de ocio, y ocupan habitaciones de hotel tras los primeros días de hospitalización.

El estado de la cuestión, los retos, problemas y repercusiones de este nuevo fenómeno son abordados en este estudio.

PALABRAS CLAVES: Intercambio sanitario, gestión, transculturalidad, competencia cultural, satisfacción, calidad cuidados, turismo sanitario.

\section{INTRODUCCIÓN / OBJETIVOS:}

El problema de las listas de espera es algo común en todos los sistemas de salud de los países del ámbito europeo. La apertura de fronteras entre los países de la Unión ha permitido el traspaso de pacientes aprovechando los diferentes recursos de los miembros con el fin de disminuirlas y así mejorar la calidad de sus servicios. Desde hace tres años la Clínica Vistahermosa de Alicante, propiedad de la compañía ASISA, viene realizando un programa en el que se interviene a este tipo de pacientes procedentes de Holanda de cirugía traumatológica. Se trata de una experiencia pionera dentro del ámbito sanitario español. La organización de este tipo de actividades de intercambio sanitario es compleja debido a todos los trámites burocráticos y socioculturales que hay que salvar (elección del país, idioma, selección de pacientes, convalidación de títulos, etc.). En cualquier caso se consigue la disminución de las listas de espera del país de origen, se desarrolla la actividad asistencial del centro privado en cuestión, se aumentan las competencias culturales del personal que participa e inevitablemente supone un impulso para el fenómeno turístico del lugar de acogida, en este caso de Alicante que ya de por sí es uno de los destinos elegidos por los europeos a la hora de disfrutar de sus vacaciones e incluso buscar una residencia fija para todo el año -como estamos viendo en los últimos años-. Es lógico pensar que estos extranjeros proceden de diferentes culturas donde parten de unos hábitos y costumbres distintos a las que se puedan dar en Alicante pero tienen la particularidad de que no sólo son turistas que acuden a pasar sus vacaciones en las costas alicantinas sino que se caracterizan porque:

- Vienen a España, concretamente a Alicante, con la finalidad de someterse a una intervención quirúrgica en la Clínica Vistahermosa a través del Proyecto sanitario "Plan de Choque" que comienza a darse entre países de la Unión Europea.

- Consecuentemente los días antes y después de la intervención realizan turismo y actividades de ocio por la provincia.

- Sus acompañantes y/o familiares también realizan estas actividades turísticas junto a ellos -es decir antes y después de la intervención- así como durante el período de ingreso del paciente. 
- Durante su ingreso en la Clínica, estos pacientes extranjeros deben adaptarse a unas normas, hábitos, costumbres...completamente distintas a las suyas propias y que ellos no eligen, como en el caso del fenómeno turístico donde son ellos los que eligen probar las costumbres o hábitos de "otros" -en este caso los alicantinos-.

Conocer estos hábitos y costumbres de personas ajenas que en un momento dado toman contacto con nuestra cultura podría abrir muchas puertas a otros trabajos de tipo político, económico y social, con la finalidad de mejorar la infraestructura de nuestra Provincia. ¿De qué forma se integran estas personas en nuestra cultura?, ¿qué es lo que les atrae de ella?, ¿qué es lo que adoptan en sus costumbres?, ¿qué es lo que conocemos de ellas?, ¿qué podemos aportarles?; estas son algunas de las cuestiones que subyacen de este discurso donde las raíces alicantinas y su interrelación con el "extranjero" se convierte en el medio primero para llegar a su respuesta. Sin embargo, en este trabajo expondremos principalmente el ESTADO DE LA CUESTION que subyace con respecto a dicho fenómeno y la LINEA DE INVESTIGACIÓN desarrollada.

\section{JUSTIFICACIÓN DEL TEMA:}

Tras un análisis de la experiencia propia de los investigadores como profesionales sanitarios, se ha observado que tras un período aceptable de tiempo, los extranjeros europeos - alemanes, noruegos y británicos en su mayoría - que se asientan en las colonias y urbanizaciones de la Costa Alicantina desarrollan una serie de respuestas sociales susceptibles del análisis antropológico. Sin embargo, actualmente asistimos a un fenómeno nuevo protagonizado por Holandeses que acuden a Alicante con la finalidad de someterse a intervenciones quirúrgicas -ya que en su país de origen las listas de espera suponen un problema social y político-. Estos holandeses aunque su principal objetivo es de tipo sanitario, se convierten en turistas una vez llegan a Alicante y no sólo ellos sino también sus acompañantes o familiares. Podríamos denominarlos por tanto como "turistas de segunda intención". A su vez resulta curioso el hecho de que estos holandeses pasan un período de tiempo hospitalizados en la Clínica Vistahermosa de Alicante en contacto con el sistema sanitario español y en contacto directo con profesionales alicantinos, dieta alicantina, costumbres, hábitos propios de la zona, etc...

Por otra parte se realizó el trabajo en la Clínica Vistahermosa por ser centro sanitario de elección en cuanto al Plan de Choque con la Unión Europea -es el centro pionero en Alicante y uno de los primeros en España- y por tratarse de uno de los centros más emblemáticos y con mayor tradición en la provincia de Alicante.

\section{MATERIAL Y METODO:}

Tras una exhaustiva búsqueda bibliográfica y documental, hemos observado que no existe ningún trabajo editado, realizado bajo las mismas condiciones del que se presenta aquí.

Se ha realizado una búsqueda bibliográfica en las bases de datos MEDLINE, CINAHL y CUIDEN. A partir de esta se puede afirmar que existe un vacío absoluto en la literatura mundial de trabajos que aborden de manera específica la forma en que los extranjeros perciben un sistema sanitario que no es el suyo, de forma práctica y todavía resulta más extraño encontrar algo en relación a lo que hemos denominado el "turismo de salud" nacido a partir del intercambio de pacientes entre países -bajo la gestión comunitaria-.

\section{DESARROLLO / ESTADO DE LA CUES- TION:}

Las listas de espera referentes a cuidados en salud no urgentes, en procesos quirúrgicos ambulatorios, así como en terapias o pruebas diagnósticas de diversa índole representan una de las problemáticas en los sistemas sanitarios públicos de países europeos tales como el sistema sanitario español, alemán, holandés o británico (MARTI, 2002). Es en algunos de estos países donde se han pretendido reducir las listas de espera empleando los servicios de las instituciones privadas, sin embargo el volumen que estos centros privados pueden soportar no es suficiente para cubrir las expectativas de los centros públicos. Otra de las estrategias políticas que han llevado a cabo algunas de estas naciones es la de trasladar a grupos de pacientes a otros centros -instituciones privadas concretamente- de países vecinos que también forman parte de la Unión Europea. Algunos países como España intentan solventar actualmente la situación problemática del 
fenómeno "lista de espera" desde dentro de sus fronteras, mientras que otros como Gran Bretaña, Alemania u Holanda contemplan en sus políticas sanitarias la opción de un Sistema de Salud fuera de sus fronteras y centrado en la reutilización de los recursos sanitarios dentro de su propio continente. Casos significativos dentro de esta problemática son como se viene diciendo los de:

- Gran Bretaña donde ya se han comenzado a realizar planes de choque sanitarios intereuropeos, favoreciendo la comparación entre sistemas sanitarios y donde se han replanteado la información al paciente y la mejora de la asistencia (BERMAN \& HIGGINS, 2002). A su vez, las políticas sanitarias contemplan el que se compartan determinadas unidades específicas -como los servicios de radiodiagnóstico y medicina nuclear- para rentabilizar el sistema (KUNKLER, 2001).

- Alemania donde algunas compañías ofertan la posibilidad de realizar intervenciones a sus asegurados fuera de sus fronteras (PAYNE, 2001).

- Holanda donde la sanidad privada no puede hacerse cargo de determinadas intervenciones y comienza su andanza dentro del fenómeno de los planes de choque sanitarios dentro de la Unión Europea (UE) en cooperación con países como Bélgica o actualmente, España.

Los órganos que conforman la Unión Europea -La Comisión Europea, el Tribunal de Justicia de la Unión Europea- se encuentran frente a una de las cuestiones de actualidad en materia de salud (HERMANS, 2000) y que se centra en la mejora de la calidad de los servicios a partir de la disminución y erradicación de dichas listas. Las reformas en las recién creadas leyes de la UE y a partir de las Leyes de Mastrich abogan por un consentimiento explícito en lo referente a planes de choque internacionales dentro de las Eurregiones - tal y como se denomina en la Comisión Europea y en el Tribunal de Justicia de la UE a los estados o naciones que conforman la UE-. Ante unas reglas que hasta el momento sólo han matizado el panorama político y económico del problema de las listas de espera, se hace necesaria una clara gestión desde todos los ámbitos de la salud y por supuesto de los cuidados que se le ofertan a los pacientes desplazados a otros centros en otros países y con culturas totalmente distintas.
Con la firma del Tratado de la Unión Europea (Maastricht, febrero 1992) y posterior reforma: el Tratado de Ámsterdam (Ámsterdam octubre 1997), se garantiza la desaparición de fronteras y la libre circulación de los ciudadanos de los países miembros de la Unión Europea.

Para ello se debe contar con la opinión de los profesionales sanitarios involucrados en el proceso de cuidar, así como de aquellos pacientes sometidos a las nuevas redes de asistencia, no únicamente desde el punto de vista diagnóstico y del tratamiento o intervención quirúrgica, sino también desde la perspectiva de la salud como algo psicosocial, procurando en todo momento la satisfacción del paciente y también del profesional autóctono.

Como se ha mostrado hasta el momento cada vez son más los europeos que reciben tratamientos médicos y atención sanitaria fuera de sus propios países de procedencia. Este fenómeno afecta a campos como el de las migraciones entre países -movimientos fuera de las fronteras-, la atención médica complementaria y los tratamientos preautorizados.

Centrando algo más el tema que a este trabajo compete, desde el año 1998 cada vez son más las clínicas privadas holandesas que han aparecido con la finalidad de hacer frente al grave problema de las listas de espera de la sanidad pública. Las leyes y la Justicia Europea han permitido esta situación siempre y cuando repercuta en el bienestar social y no se perjudique al paciente, es decir, que los centros sean de calidad para el mismo (BROUWER \& HERMANS, 1999).

Actualmente en España nos encontramos ante el primer caso de Plan de Choque sanitario de un país de la UE como es el caso de Holanda. Desde hace dos años la Clínica Vistahermosa de Alicante, propiedad de la compañía ASISA, viene realizando un programa en el que se intervienen a este tipo de pacientes procedentes de Holanda de cirugía traumatológica. Se trata de una experiencia pionera dentro del ámbito sanitario español. Hasta el momento parece que esta nueva modalidad de atención internacional y dentro de la UE sólo se ha caracterizado por su efectividad a la hora de reducir el problema de las listas de espera de los países que la conforman, sin embargo este objetivo parece haber eclipsado otros como el de la satisfacción del paciente ante el nuevo sistema sanitario o la com- 
petencia del profesional ante pacientes de otras culturas. Actualmente son 148 los pacientes holandeses pertenecientes al plan de choque que durante dos años han pasado por las instalaciones de la Clínica Vistahermosa y se han observado situaciones comprometidas derivadas del choque cultural que se ha dado en cuanto a las necesidades básicas y ámbitos de la vida cotidiana (LILLO, 2003c). Los profesionales sanitarios de los cuidados se ven obligados a tratar con estos pacientes sin conocer las características de su cultura de procedencia, así como el mismo paciente quien debe adaptarse a hábitos, costumbres y situaciones completamente desconocidas para él.

Los últimos análisis de las tendencias migratorias en diversos países de la Comunidad Europea (OCDE, 1998) y en España (PAJARES, 1998; Instituto de Estudios Transnacionales, 1997) alertan sobre la necesidad de desarrollar políticas adecuadas para hacer frente a las nuevas situaciones, de forma integral, de colectivos de inmigrantes que van a necesitar un importante apoyo educativo y sociosanitario en el que la enfermería transcultural debe desempeñar un papel de primer orden. Uno de los problemas que acechan de forma especialmente preocupante en este contexto inmigratorio es el conflicto intercultural de forma que se deben activar las conciencias para no acabar practicando una enfermería parcelada por grupos culturales (BARBADILLO, 1997; CALVO, 1997; ESPADA, 1997; VV.AA., 1997). Sin duda alguna la sociedad española y también la europea debe prepararse para una convivencia plural. Pero la convivencia multicultural implica cuidados especiales (KRISTEVA, 1991).

España, país que se presenta a la recién estrenada Unión Europea como una nación de servicios y donde el turismo parece ser una de sus principales fuentes de ingresos se enfrenta actualmente a un nuevo fenómeno sociocultural: "los planes de choque intereuropeos". Los sistemas sanitarios españoles llevan años haciendo frente a una realidad que ha ido en alza como es la presencia de extranjeros en el sistema sanitario español, de hecho mas de la mitad de municipios con un $25 \%$ de extranjeros esta en la provincia de Alicante. Sin embargo los servicios de salud todavía continúan trabajando de la misma forma y no existen protocolos, publicaciones ni siquiera se han hecho nuevos plantea- mientos acerca de dicha diversidad cultural en la comunidad científica. Los estudios pasan por ser principalmente poblacionales y demográficos y en su defecto tocan el tema de la Inmigración desde la perspectiva del Trabajo Social. Un estudio del INE desvela que los principales destinos que la población extranjera elige para su residencia son, en líneas generales, poblaciones turísticas localizadas en el Mediterráneo o Canarias -con británicos y alemanes en su mayoría- poblaciones que necesitan mano de obra agrícola, ubicadas fundamentalmente en Almería y Murcia -marroquíes, predominantemente- y pequeñas poblaciones del interior, donde la minoría extranjera esta en función de la primera comunidad que se asienta allí. En los últimos años, en toda la costa mediterránea española, ha habido un aumento muy importante de la población del centro y del norte de Europa tanto como residentes durante todo el año como de no residentes, es decir, turistas o que ocupan un período vacacional (y que también son susceptibles de ingreso hospitalario). En concreto en la provincia de Alicante encontramos grupos numerosos de escandinavos en la comarca de la Marina Alta (sobre todo en Alfaz del Pí) e ingleses en Torrevieja. El grupo que nos ocupa comprende a holandeses en su mayoría. Estos grupos de población suelen estar jubilados y la edad media está por encima de los 60 años. Todo esto tiene como consecuencia que el número de hospitalizaciones de estas personas vaya en aumento. Para este grupo, la costa levantina se encuentra entre sus preferencias a la hora de disfrutar de sus vacaciones, por lo que también el número de hospitalizaciones de no residentes es considerable (LILLO, 2003c).

Como se demuestra el sistema sanitario alicantino se encuentra mas que acostumbrado a trabajar con usuarios procedentes de culturas totalmente diferentes y sin embargo todavía no se ha puesto manos a la obra en cuanto al estudio de la satisfacción de este tipo de pacientes, su calidad, sensibilidad cultural de los profesionales que les atienden, competencia cultural, así como el universo de los valores y creencias.

Resumiendo podemos decir que no existe ningún otro trabajo en los catálogos bibliográficos que trate a los pacientes de los planes de choque intereuropeos desde la perspectiva de la Enfermería Transcultural y concretamente desde temáticas 
como la satisfacción o la competencia cultural. El fenómeno de los planes de choque entre las Eurregiones solamente aparece reflejado en la literatura como un problema político, legal y económico o simplemente como un hecho descrito pero no analizado específicamente (MARTI, 2002; BERMAN \& HIGGINS, 2002; PAYNE, 2001; HERMANS, 2000; BROUWER \& HERMANS, 1999).

\section{LINEA DE INVESTIGACIÓN:}

La línea de trabajo en la cual se ha enfocado dicho fenómeno a estudio se propone los siguientes objetivos:

- Analizar el choque cultural que se produce entre los hábitos y costumbres - es decir, las repercusiones socioculturales- de los extranjeros no residentes en Alicante que proceden de los países de la Unión Europea incluidos en el proyecto sanitario "Plan de choque europeo" -centrándonos tanto en el paciente como en el acompañante / familia- y los hábitos y costumbres propios de Alicante, así como los específicos de su sistema sanitario.

- Conocer las características en cuanto al fenómeno ocio y turismo respecto de los extranjeros -tanto en el caso del paciente como el acompañante / familia- hacia Alicante, es decir: expectativas con las que llegan a Alicante, imagen que tenían -tanto de la ciudad y provincia como de su ámbito sanitario-, tipo de turismo que realizan, actividades y ocio que prefieren desarrollar, imagen con la que vuelven a su país de origen...

\section{BIBLIOGRAFÍA:}

- AUGE, M. (1992). Los no lugares. Espacios del anonimato. Una antropología de la sobremodernidad. Barcelona: Antropología, Gedisa ed.

- AVIS, M. (1995). Satisfying solutions? A review of some unresolved issues in the measurement of patient satisfaction. Journal of Advanced Nursing, 22(2),316-322.

- BARBADILLO, P. (1997). Extranjería, racismo y xenofobia en la España contemporánea. La evolución de los setenta a los noventa. Madrid: Centro de Investigaciones Sociológicas/ Siglo XXI de España editores, colección monografías.

- BERMAN, P.; HIGGINS, J. (2002). Treating pacients abroad. Worlds apart. Health Services Journal, 112 (5787): 24-26.

- BOND, S., THOMAS, L.H.(1992). Measuring patients' satisfaction with nursing care. Journal of advanced nursing, 17, 5263.

- BOYLE, J. (1994). Styles of ethnography. En MORSE, JM. (Ed.), Qualitative health research. Newbury Park, CA: Sage. - BROUWER, WB.; HERMANS, HE. (1999). Private Clinics for employees as a Dutch solution for waiting lists: economic and legal arguments. Health Policy, 47(1):1-17.
- CAMPBELL, I.E., LARRIVEE, L., FIELD, P.A., DAY, R.A., REUTTER, L. (1994). Learning to nurse in the clinical setting. Journal of Advanced Nursing, 20, 1125-1131.

- CALVO, T. (1997). Racismo y solidaridad de españoles, portugueses y latinoamericanos. Los jóvenes ante otros pueblos y culturas. Madrid:Ediciones Libertarias.

- CASABONA, I.; LILlO, M.; GALAO, R.; MORA, MD. (2003). Diferencias culturales en una Unidad de Cuidados Críticos. Aceptado para su publicación 2003 Revista MONITOR de la Asociación de Enfermería en Anestesia, Reanimación y Terapia del Dolor de la Comunidad Valenciana.

- CASABONA, I.; LILlO, M.; GALAO, R.; MORA, MD. (2003). Unidad de Cuidados criticos: implicaciones culturales. Alcira: Ponencia pronunciada durante las Jornadas ASECVAR 2003 en la mesa debate, Hospital de la Ribera, Alcira.

- CRIVELLI, L.; ZWEIFEL, P. (1998). Modelling cross-border care in the EU using a principle agent framework. Dev Health Econ Public Policy, 6: 229-257.

- CUERVO, J.; VARELA, L.; BELENES, R. (1994). Gestión de Hospitales: Nuevos instrumentos y tendencias. Barcelona: Ed. Vicens Vives.

- DEAN, M. (2001). Alternatives to treatment in the UK. Lancet, 358(9280):479.

- ESPADA, ML. (1997). ¿Europa, ciudad abierta? La inmigración y el asilo en la Unión Europea. Granada: Instituto Municipal de formación y empleo.

- FONG, CM. (1985). Ethnicity and nursing practice. Topics in Clinical Nursing, 7(3):1-10.

- GARCIA, JL. (1995). Como elaborar un proyecto de investigación. Alicante: Publicaciones de la Universidad de Alicante. - HERMANS, HE. (2000). Cross-border health care in the European Union: recent legal implications of "Decker and Kholl". Journal of Evaluation of Clinical Practice, 6(4): 431439.

- INSTITUTO DE ESTUDIOS TRANSNACIONALES (1997). Mediterráneo, ¿camino abierto o frontera?. Cordoba: INET.

- KENNY, C. (2001). European Health care. Vive la difference?. Nursing Times, 97(42):12-13.

- KRISTEVA, J. (1991). Extranjeros para nosotros mismos: ¿Sera posible la convivencia multirracial en la Europa del siglo XX?. Barcelona: Plaza \& Janes.

- KUNKLER, I. (2001). Radiotherapy units should be seen as shared resource in EU. BMJ, 323 (7319):1004.

- LEININGER, M. (1995). Transcultural nursing: Concepts, Theories, Research and Practices. Nueva York: McGraw-Hill ( $2^{\text {a }}$ edición).

- LEININGER, M. (1999). Cuidar a los que son de culturas diferentes requiere el conocimiento y las aptitudes de la Enfermería Transcultural. Revista de Enfermería y Humanidades Cultura de los Cuidados, 6(3), 5-12.

- LILLO, M (2001). Diario de Campo: Las tribulaciones de un participante observador. Revista INDEX de Enfermería, 10 (35).

- LILLO, M. (2002a). Antropología de los cuidados en el anciano: evolución de los valores sociales sobre la vejez. Buenos Aires: CDROM Sociedad NAYA Congreso Virtual 2002.

- LILLO, M. (2002b). Subculturas de Identidad en el ámbito sanitario: Enfermería en Intensivos. Buenos Aires: CDROM Sociedad NAYA Congreso Virtual 2002. 
- LILLO, M. (2002c). Antropología, género y enfermería contemporánea. Revista ROL de Enfermería. 25 (12), 56-62.

- LILLO, M; VIZCAYA, F. (2002). Origen y desarrollo de los hábitos y costumbres alimentarias como recurso sociocultural del ser humano: Una aproximación a la Historia y Antropología de los cuidados en la Alimentación. Revista de Enfermería y Humanidades Cultura de los Cuidados 11(6), 6165.

- LILlO, M.; CASABONA, I.; GALAO, R.; MORA, MD. (2003a). Enfermería Transcultural en el contexto de la práctica clínica. 3er Premio de Investigación CECOVA. Revista de la Comisión de Investigación del Colegio de Enfermería de Alicante, 1, 1-6.

- LILLO, M.; VIZCAYA, F.; DOMÍNGUEZ, M.; GALAO, R. (2003b). Investigación cualitativa en Enfermería y Competencia Cultural. Aceptado para su publicación 2003 Revista INDEX de Enfermería.

- LILlO, M.; CASABONA, I. (2003c). El profesional de Enfermería en Cuidados Críticos: subculturas de identidad en el ámbito sanitario. Aceptado para su publicación 2003 Revista MONITOR.

- LILLO, M.; VIZCAYA, F; DOMÍNGUEZ, M; FERRER, R. (2003d). Síntomas manifestados por mujeres con angina de pecho: un estudio transcultural e internacional. Revista MONITOR de la Asociación de Anestesia, Reanimación y Terapia del Dolor de la Comunidad Valenciana, 4, 19-21.

- LILlO, M.; CASABONA, I.; MORA, MD.; GALAO, R. (2003e). La imagen de la Enfermería española en Cuidados críticos vista por los pacientes centroeuropeos. 1er Premio ASECVAR-TD 2003. Comunicación a las Jornadas ASECVAR-TD/ Hospital de la Ribera (Alcira) 2003. Aceptado para su publicación 2003 en Revista MONITOR.
- LIPSON, JG.; MELEIS, AI. (1989). Methodological issues in research with immigrants. Medical Anthropology, 12:103-115.

- MARTI, J. (2002). La gestión de las listas de espera quirúrgicas por los centros y los profesionales. Gaceta Sanitaria, 16(5): 440-443.

- MERKOURIS, A. (1999). Patient satisfaction: a key concept for evaluating and improving nursing services. Journal of Nursing Management, 7(1):19-28.

- MORIN, E. (1984). Ciencia con consciencia. Barcelona: Anthropos, Editorial del Hombre.

- MORSE, J. (1989). Qualitative Nursing Research. A Contemporary Dialogue. London: Sage Publications.

- MORSE, J. (1992). Qualitative health research. Newbury Park, CA: Sage.

- MORSE, J.; FIELD, PA. (1995). Qualitative research methods for health professionals. Thousand Oaks, CA: Sage.

- MURDOCK, G. (1971). Outline of cultural materials. New Haven: Human Relations Area Files.

- OCDE. (1998). Tendances des migrations internationales. Paris: Rapport annuel, OCDE.

- PAIGE, MR. (1986). Cross-cultural orientation: New conceptualizations and applications. Lanham, MD: University Press of America.

- PAJARES, M. (1998). La inmigración en España. Madrid: Icaria.

- PAYNE, D. (2001). German company offers "package deal ops" to Ireland. BMJ, 323(7311): 471.

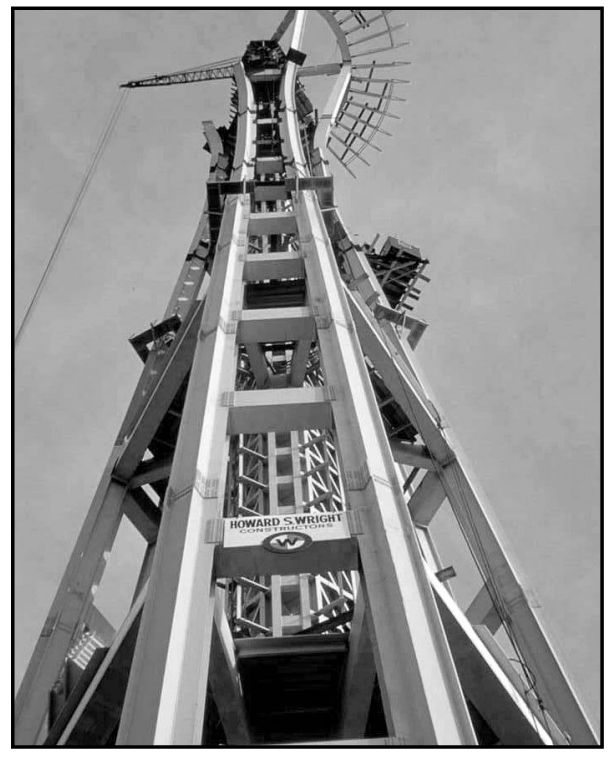

